NOUVELle

\section{Difficile cohabitation entre hyènes des cavernes et hommes de Néandertal il y a 40000 ans}

Cédric Beauval
Archéosphère, Domaine du Haut-

Carré, 351, cours de la Libération, 33405 Talence Cedex, France. PACEA, UMR 5199, Université Bordeaux 1, avenue des Facultés, 33505 Talence Cedex, France.

c.beauval@ipgq.u-bordeauxl.fr
> En cinq ans, le site archéologique des Rochers de Villeneuve (Lussac-les-Châteaux, Vienne, France) a livré plus de 130000 vestiges témoignant de l'occupation de la grotte par les hyènes des cavernes et les hommes de Néandertal. Parmi ces restes, un fragment de fémur humain (Figure l) présente à ses deux extrémités des traces de dents d'un grand carnivore. Les hommes de Néandertal, comme les australopithèques et les Homo erectus, semblent donc apparaître au menu des grands carnivores préhistoriques. Cette petite grotte $(10 \mathrm{~m}$ de long, $7 \mathrm{~m}$ de large) s'ouvre au sommet d'une petite falaise, à une vingtaine de mètres au-dessus d'un affluent de la Vienne (Figure 2). Un grand plateau calcaire s'étend vers le nord, limité à l'ouest et à l'est par les vallées de la Vienne et de la Gartempe. La cavité est connue des préhistoriens depuis 1969. Cette année-là, Pierre Boutin et André Chollet mettent en évidence un niveau archéologique livrant du bison, du cheval, de la hyène des cavernes et quelques silex. Une fouille programmée du site conduite par Cédric Beauval et financée par le Ministère de la Culture débute en 1999. L'objectif de cette fouille est d'apporter de nouveaux éléments pour décrire le mode de vie des hyènes préhistoriques et d'appréhender les relations entre ces grands carnivores et un autre redoutable prédateur: I'homme de Néandertal. Les premières campagnes de fouilles confirment les observations des recherches antérieures: hommes et hyènes ont fréquenté le site et $y$ ont abandonné les déchets de leurs repas et de leurs productions de silex. Tous ces restes sont découverts dans un même niveau archéologique, et il est délicat de dissocier ce qui est apporté par les hommes de ce qui est accumulé par les carnivores. En effet, l'ensemble archéologique a subi des conditions périglaciaires et les alternances gel-dégel ont provoqué une migration des vestiges au sein de la matrice sédimentaire. Ces processus affectent surtout les objets les plus volumineux; ces derniers ont été expulsés à la surface du sol alors que les objets plus petits ont été plus ou moins déplacés en fonction de leur taille. Cependant, quelques os permettent d'avancer l'ébauche d'un scénario. En effet, des éléments d'une carcasse de cheval et un fragment de fémur de bison présentent à la fois des traces laissées par le passage du tranchant d'un silex et des traces de dents de hyènes. Les stries de silex caractérisent la désarticulation

des vertèbres et des côtes pour le cheval, et celle du fémur et du tibia pour le bison. Les traces de dents sont observées à chaque extrémité des fragments osseux et l'une d'entre elles recoupe une strie sur une vertèbre de cheval. L'exploitation de ces os par les carnivores est donc postérieure à celle réalisée par les hommes. De plus, puisque les restes abandonnés par les hommes présentent encore un intérêt nutritif pour les hyènes, les fréquentations humaines et animales de la grotte sont contemporaines à l'échelle archéologique. Une datation au carbone 14 réalisée sur un os de hyène situe cet épisode il y a environ 40000 ans.

En juin 2002, Charlène Bouyssou, étudiante en histoire de l'art et archéologie à l'Université de Poitiers (France), dégage du niveau archéologique un cylindre osseux de $20 \mathrm{~cm}$ de longueur. Cette pièce a une section circulaire; elle présente une ligne âpre bien visible et légèrement en relief malgré l'absence

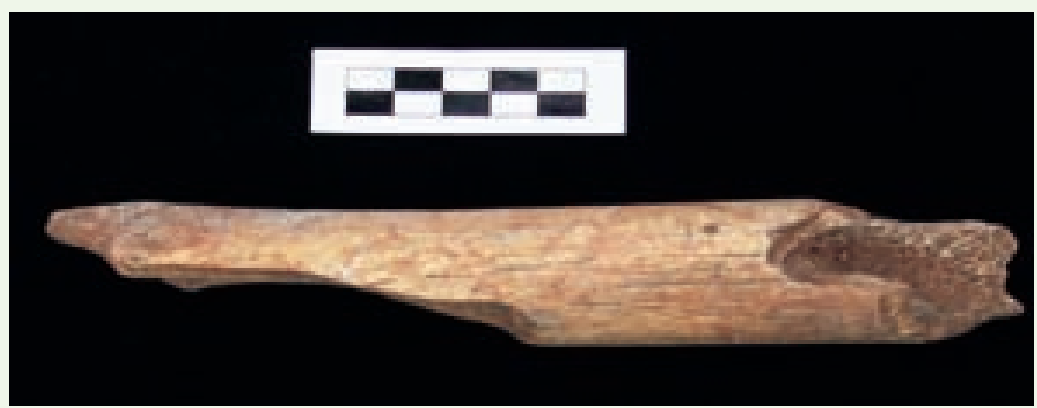

Figure 1. Fragment de fémur humain présentant à ses deux éxtremités des traces de dents d'un grand carnivore. Échelle (rectangles blancs et noirs) : $1 \mathrm{~cm}$. 
de pilastre (pilier osseux). Cette morphologie est celle d'un fémur humain. Elle s'inscrit parfaitement dans la variabilité des fémurs néandertaliens alors qu'elle s'éloigne de la morphologie des homologues de l'homme moderne. L'os possède les dimensions d'un os mature, mais en l'absence des épiphyses, l'âge de l'individu ne peut être précisé. II est fragmenté aux deux extrémités. À proximité de ces fractures, de petites dépressions circulaires dans la surface corticale témoignent du mâchonnement de la diaphyse par un grand carnivore.
Aucun autre stigmate et aucune pathologie ne sont observables sur le fragment. Un échantillon de $300 \mathrm{mg}$ a été prélevé pour effectuer une analyse d'ADN. Cette étude, réalisée par David Serre (Max Planck Institute, Leipzig, Allemagne), a permis d'isoler une séquence de $31 \mathrm{pb}$, identique à celle des fossiles néandertaliens de La Chapelle-aux-Saints 1, Mezmaiskaya 1, Feldhofer 2 et Engis 2. Elle diffère de celle des autres néandertaliens connus par 1 pb et de celle des hommes modernes par 3 pb. La conservation de I'ADN n'a pas permis d'isoler de

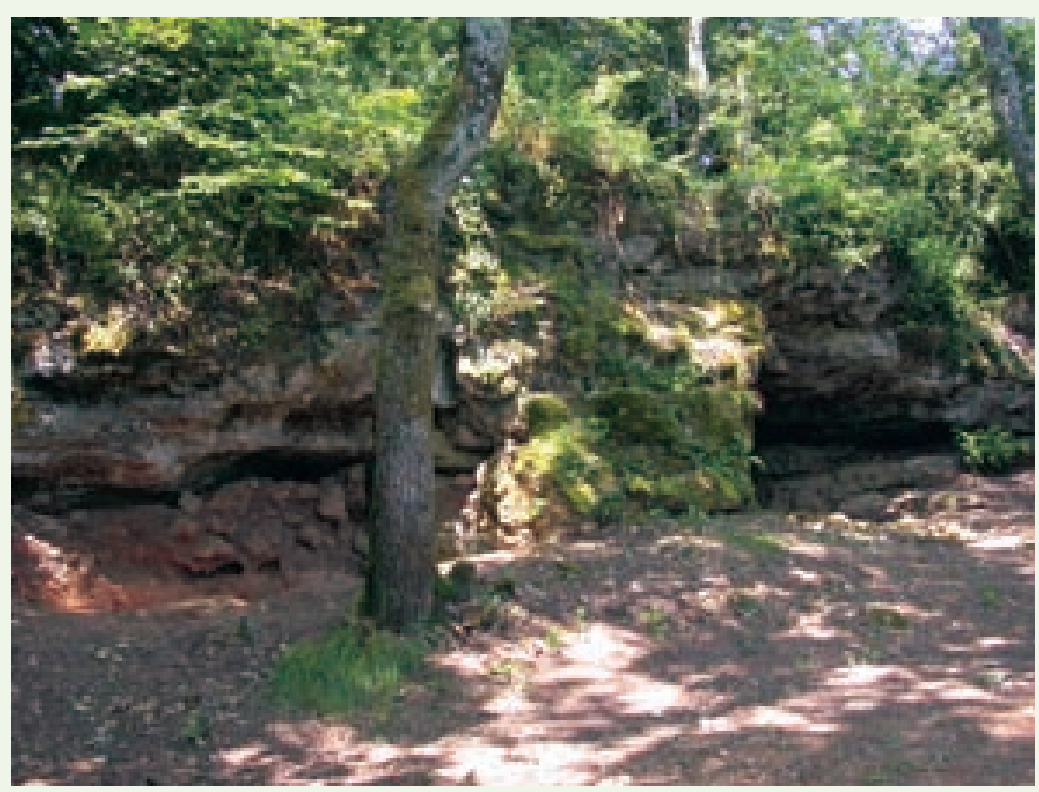

Figure 2. Site archéologique des Rochers de Villeneuve (Lussac-les-Châteaux, Vienne).

séquence supplémentaire. Le contexte archéologique, l'étude morphométrique et l'analyse génétique de ce vestige plaide donc pour une détermination de la pièce comme néandertalienne [1]. La datation du niveau en fait l'un des restes les plus récents de cette lignée. Par ailleurs, le renforcement antéropostérieur de la diaphyse constaté sur la diaphyse des Rochers de Villeneuve le rapproche du fossile de la Roche-àPierrot [2], découvert dans un niveau châtelperronien (première culture du Paléolithique supérieur, vers 30000 ans). Cette modification de la morphologie de la diaphyse fémorale chez les derniers néandertaliens pourrait être mise en relation avec la mobilité plus importante des hommes à la fin du Paléolithique moyen et au cours du Paléolithique supérieur. Ce trait morphologique appuie la thèse d'un changement biocomportemental important au sein de la lignée humaine en Europe quelques millénaires avant l'arrivée des premiers hommes modernes. $\diamond$

A late Neandertal femur from Les Rochers-de-Villeneuve, France

\section{RéFÉRENCES}

1. Beauval C, Maureille B, Lacrampe-Cuyaubere F, et al. A late Neandertal femur from Les Rochers-deVilleneuve, France. Proc Natl Acad Sci USA 2005 ; $102: 7085-90$.

2. Trinkaus $\varepsilon$. Long bone schaft robusticity and body proportions of the Saint-Cesaire 1 chatelperronian Neanderthal. J Archaeol Sci 1999 ; 26 : 753-73.

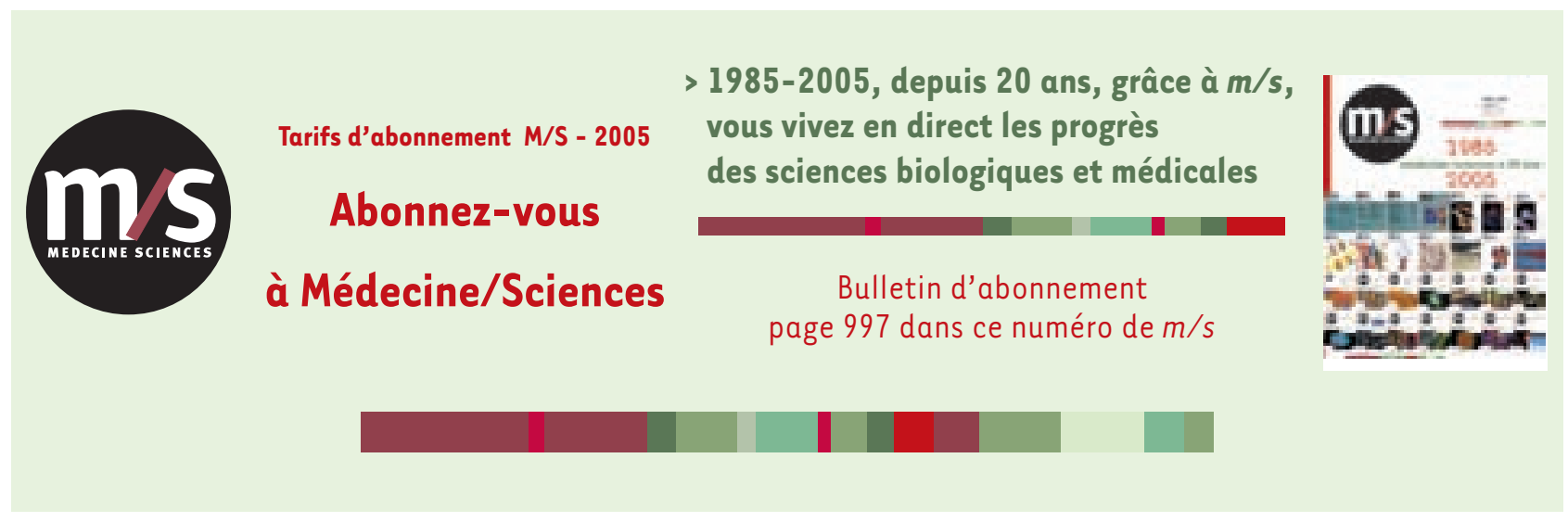

